\title{
Bootstrap assessment of the reliability of maxima in surface maps of brain activity of individual subjects derived with electrophysiological and optical methods
}

\author{
MONICA FABIANI and GABRIELE GRATTON \\ University of Missouri, Columbia, Missouri \\ PAUL M. CORBALLIS \\ Dartmouth College, Hanover, New Hampshire \\ and \\ JEFF CHENG and DAVID FRIEDMAN \\ New York State Psychiatric Institute, New York, New York
}

\begin{abstract}
Surface maps of brain activity can be obtained with electrophysiological and optical recordings. However, there are no established methods for determining the reliability of maps of brain activity across subject groups or across tasks within the same subject. In this paper, we use bootstrapping to establish the reliability of the locations of maxima in maps of surface brain activity of individual subjects obtained with ERP and optical (EROS) recordings and report sample analyses for two data sets. Bootstrapping is a nonparametric method for estimating statistical accuracy from the data in a single sample. The distribution of the statistic of interest is estimated by constructing "bootstrap samples" from a pool of all available cases (with replacement). Many "bootstrap replications" are obtained by calculating the statistic of interest for each sample. In the case of brain activity, many (e.g., 10,000) amplitude distributions can be derived from the data of an individual subject. Frequency counts are then computed for each recording location to establish how many times that location corresponds to a maximum. The value obtained in this fashion represents an estimate of the reliability of the observation.
\end{abstract}

The last decade has seen an enormous increase in the attention that is paid to the relative contributions of various areas of the brain to perception, cognition, and movement planning and execution. This has been accompanied by the development and/or increased use of several noninvasive brain imaging techniques, such as PET, fMRI, and, more recently, transcranial optical imaging (Toga \& Mazziotta, 1996). In electrophysiology, thanks also to electronic and computational advances, this emphasis has corresponded to a large increase in the number of scalp locations used to record event-related brain potentials (ERPs) and the magnetoencephalogram (MEG).

When ERP data are collected from dense electrode arrays, surface maps of the scalp distribution of potentials can be generated (e.g., Duffy, 1982; Gevins, 1996). One of the main uses of these maps is to examine possible dif-

The research reported in this paper was supported by Grant AG05213 from the National Institute of Aging to D.F. and by NIMH Grant 5R01MH57125-01 to G.G. A preliminary report of these data was presented at the 35th Annual Meeting of the Society for Psychophysiological Research, Toronto, Canada. Correspondence should be addressed to M. Fabiani, Department of Psychology, University of Missouri, 210 McAlester Hall, Columbia, MO 65211-2500 (e-mail: psymf@showme.missouri.edu). ferences in the surface localization of brain potentials at a glance, assuming that different intracranial generators and/or different cognitive processes may be active when scalp distribution differences are observed.

One of the features of surface maps that researchers pay attention to is the location of foci of maximum activity, either within a time window that may encompass an entire ERP component (such as P3) or within a smaller segment of the average waveform. For example, investigators may focus on a change in the location of the maximum of the P3 component from a frontal to a parietal focus, over the course of a task, or for different groups of subjects (e.g., Fabiani \& Friedman, 1995; Friedman, Simpson, \& Hamberger, 1993).

Because of this increased emphasis on scalp distribution, the issue of the within-subject reliability of some of the scalp distribution features (such as the location of maxima) becomes important. However, there are no established methods for assessing the reliability of these features in individual subjects. A major problem is that assumptions about the normality of the distribution of values observed at each electrode are often violated. This may limit the validity of parametric techniques. Some investigators (e.g., Karniski, Blair, \& Snider, 1994) have proposed the use of distribution-free approaches in the analysis of scalp 
distribution data. In this paper, we propose the use of "bootstrapping" to address the issue of the reliability of the location of maxima.

The bootstrap is a nonparametric method for studying the distribution of sample statistics (Diaconis \& Efron, 1983; Efron, 1979; Efron \& Gong, 1983; Efron \& Tibshirani, 1985). It is based on an empirical (rather than theoretical) approach to the estimate of population parameters, since it makes no a priori assumptions about the data distribution. In this sense, it is similar to the jackknife, cross-validation, and Monte Carlo approaches, in that it replaces standard assumptions with massive calculations. Bootstrapping has been applied in several fields, including evolutionary biology and population genetics (e.g., Brown, 1994; Chiano \& Yates, 1994). The use of bootstrapping in electrophysiology was described by Wasserman and Bockenholt (1989), and this procedure has been applied in a number of cases (e.g., Fabiani, Karis, \& Donchin, 1986; Farwell \& Donchin, 1988; Karis, Fabiani, \& Donchin, 1984). The aim of this paper is to propose the use of bootstrapping for the specific purpose of analyzing the reliability of maxima in surface maps of brain activity of individual subjects and to review in detail the steps that this procedure requires. It is important to keep in mind, however, that the bootstrap is a very flexible method that can be adapted to a number of statistical testing purposes.

Bootstrapping involves drawing many "bootstrap samples" (with replacement, i.e., with the possibility that the same case could be used more than once within the same bootstrap sample) from the empirical data. This is achieved by duplicating each case in the sample a very large number of times and then by drawing, at random, many new samples from this enlarged database. Accordingly, a given bootstrap sample may be composed of cases that are all unique (and, therefore, it would be identical to the original sample), or, at the other end of the spectrum, another bootstrap sample may be composed of many duplications of the very same case. Thus, it is fundamental (as in all similar randomization procedures) that enough bootstrap samples be created to achieve a representative distribution of these alternative possibilities.

Once the pool of random bootstrap samples has been established, the next step is to calculate the statistic of interest (e.g., mean value, correlation, frequency count of a given attribute, etc.) for each sample ("bootstrap replications"). At this point, a bootstrap distribution can be created from the bootstrap replications, and this will provide an estimate of the variability of the statistic of interest.

There are several reasons why the bootstrap may be advantageous (with respect to parametric approaches) in the estimation of the reliability of brain activity. First, parametric statistics usually make strong assumptions about the data distribution. However, these assumptions are difficult to verify and are often violated, especially in small samples. There are a number of cases in which research involving the recording of brain activity in humans is limited to small samples. For example, there is a tradeoff between statistical accuracy and the cost (both in terms of time and money) of running a large number of subjects and/or trials. Also, subjects from some special populations are rare, and, therefore, large samples are simply not available. Finally, there may be limitations that are inherent to specific experimental paradigms or procedures.

In the remainder of this paper, the procedures used to perform a bootstrap analysis are described in detail for two examples based on actual data. The first example involves the bootstrapping of electrophysiological (ERP) data recorded from 30 scalp electrodes. The second example involves the bootstrapping of optical brain imaging data recorded from 12 scalp locations. Finally, analyses and results are discussed. Two rather similar examples are described since they refer to two different techniques that vary on the basis of the correlation that can be observed between adjacent recording locations. In fact, the ERP is a well-established method with limited localization power and low spatial frequency (due to volume conduction), whereas optical imaging is a newer technique with greater localization power and higher spatial frequency. It is important to show that bootstrap methods provide useful information in both cases and that they are therefore generalizable to a variety of brain mapping data.

\section{EXAMPLE 1 \\ Reliability of Maxima in ERP Maps of Brain Activity}

It is well known that the P3 component of the ERP is often reduced in amplitude (relative to controls) in several different subject populations, including depressed and schizophrenic patients (Diner, Holcomb, \& Dykman, 1985; Pfefferbaum, Wenegrat, Ford, Roth, \& Kopell, 1984) and older subjects (e.g., Polich, 1991; Polich \& Starr, 1984). A difference in scalp distribution of the P3 is also commonly observed between young and old adult subjects (e.g., Ford \& Pfefferbaum, 1985; Friedman, 1995; Friedman \& Fabiani, 1995). Young subjects usually have $\mathrm{P} 3 \mathrm{~s}$ that are maximum at the posterior scalp (parietal electrodes), whereas older adults tend to have P3s that are more equipotential across electrodes and sometimes larger at frontal recording sites.

If one wants to compare these different populations, the question of within-subject reliability becomes important. In fact, a reduced $\mathrm{P} 3$ amplitude can lead to reduced stability of the spatial location of peaks of maximum P3 activity, with consequent instability in the reliability of differences in scalp distribution, and it can possibly confound the results. In this example, we demonstrate the use of the bootstrap in the assessment of the reliability of maxima for individual subjects' waveforms.

\section{Method}

Recording procedures. The data for this example were selected from those of a larger study focused on the relationship between scalp distribution differences in aging and neuropsychological variables, which will be published separately (Fabiani, Friedman, \& Cheng, 1998). Four young and 4 old female subjects were selected from the larger subject sample to be representative of the range of variation of their respective groups. The data were collected in a novelty oddball 
paradigm, in which the subjects heard a random series of 400 sounds, at a rate of 1 per second. The sounds used in the series consisted of two pure tones (one high $=500 \mathrm{~Hz}$, and the other low $=250 \mathrm{~Hz}$ ) repeated many times and of a number of unique nontonal sounds (novel stimuli). The probabilities of occurrence of the different types of sounds were as follows: For each subject, one of the two tones was designated as the rare target ( $p=.12 ; 48$ stimuli), and the other was designated as the frequent standard $(p=.76)$, whereas the novel stimuli occurred with a probability of .12 . The subjects were instructed to respond to the target tones and to withhold their response to the standard stimuli (go/no-go procedure). The novel sounds, which occurred unexpectedly, also required no response. The responding hand and the target tone (high or low) were counterbalanced across subjects. The bootstrap analysis presented here is limited to the target stimuli, for which a P3 is expected to be evident, and to reflect the typical age-related differences in distribution.

Electroencephalographic (EEG) activity was recorded from 30 placements (referred to the nosetip) by means of an electrode cap (Electrocap International, Inc.) for the sites located on the scalp and by means of disposable $\mathrm{Ag} / \mathrm{AgCl}$ electrodes for sites located on the face and mastoids. A schematic representation of the electrode sites is presented in Figure 1.

Horizontal and vertical electrooculographic (EOG) activity was recorded bipolarly from electrodes located above and below the right eye and at the outer canthi of both eyes, respectively. The EOG and EEG were recorded with a $30-\mathrm{Hz}$ high-frequency filter and a 5.3 -sec time constant and were digitized at 200 samples per second. Eye-movement artifacts were corrected off line by means of a procedure developed by G. Gratton, Coles, and Donchin (1983). In addition, single trials were visually inspected, and trials containing muscular and/or other recording artifacts were marked and excluded from all further analyses.

Step 1: Creating the bootstrap samples. As outlined in the introduction, the first step of the bootstrap procedure involves the creation of many bootstrap samples that are made by duplicating a very large number of times the data of each of the subjects contained in the original sample. Many new samples are then drawn (at random and with replacement) from this expanded trial pool. In this exam- ple, both this analysis and the following analyses were conducted within subjects (i.e., there was no pooling across subjects). For examples of the use of bootstrap in ERP research applied to a between-subjects case, see Karis et al. (1984) and Srebro (1996).

If the interest is (as in this case) to establish the reliability of the scalp locations of the largest amplitude target $P 3$ of each individual subject, an alternative procedure is also possible, which is equivalent to the one just described from the mathematical point of view but makes the programming and calculations considerably easier. First, for each subject, mean amplitude measures of the target P3 were obtained separately for each trial and electrode. These measures were taken with respect to a prestimulus baseline, within a time window of 250-435 msec poststimulus, which could encompass the P3 component for both young and old subjects. Thus, for each subject, the resulting data set consisted of samples of singletrial P3 mean amplitude measures for each of the target stimuli and each of the 30 electrode sites.

For each subject's data set, a very large number of bootstrap samples of trials was then randomly selected from the available pool of trials (with replacement). In the example presented here, 10,000 such samples were created for each subject. The maximum number of target trials available for each subject was 48 . Therefore, samples of 48 trials were used for constructing the bootstrap samples. A schematic representation of this procedure is presented in Figure 2.

Step 2: Calculation of the statistic of interest for each bootstrap sample (the bootstrap replications). For each subject and bootstrap sample, a new average measure of the target P3 amplitude was computed for each electrode site, and then the site of maximum amplitude (out of the 30 electrode sites) was identified

Step 3: Generating the bootstrap distribution. At this point, the number of times (i.e., frequency count) that the target $P 3$ was largest at any given electrode site was calculated, and the distribution of these frequency counts was examined. If most of the time (e.g., 9,500/10,000, $p \leq .05$ ) the target $\mathrm{P} 3$ maximum was located at one particular electrode site, the distribution of the peak could be considered very reliable. Even if the maxima were distributed across various locations, it is of interest to examine whether the high counts clustered at locations that are adjacent to each other.

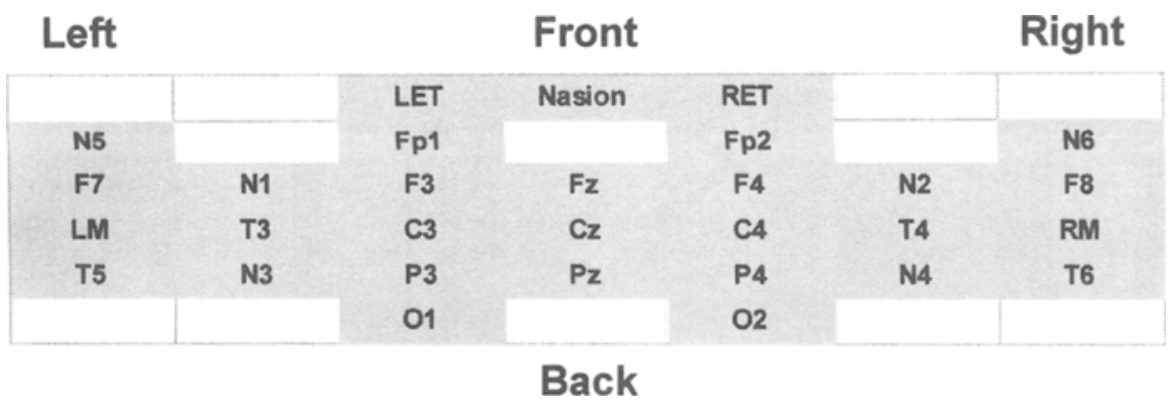

Figure 1. Schematic representation of the electrode montage used for Example 1. The following electrodes were placed according to the standard 10-20 System: $\mathrm{Fz}, \mathrm{Cz}, \mathrm{Pz}, \mathrm{F7}, \mathrm{F8}, \mathrm{T3}, \mathrm{T4}$, T5, T6, O1, and $O 2$, right and left mastoid. Nonstandard electrode placements were located as follows: Fp1', $16 \%$ in front of $\mathrm{Fz}$ on the midline and $10 \%$ laterally on the left hemisphere; Fp2', homologous of $\mathrm{Fp}^{\prime}$ on the right hemisphere; $\mathrm{F3}^{\prime}, 33 \%$ of the distance on a line between $\mathrm{Cz}$ and $\mathrm{F3}$, on the left hemisphere, closer to $\mathrm{F3}^{\prime}$; $\mathrm{F4}^{\prime}$, homologous of $\mathrm{F3}^{\prime}$ on the right hemisphere; $\mathrm{C3}^{\prime}$, $60 \%$ of the distance on a line between $\mathrm{Cz}$ and $\mathrm{C3}$, on the left hemisphere, closer to $\mathrm{C} 3$; $\mathrm{C4}^{\prime}$, homologous of $\mathrm{C3}^{\prime}$ on the right hemisphere; $\mathrm{P}^{\prime}, 65 \%$ of the distance on a line between $\mathrm{Pz}$ and $\mathrm{P3}$, on the left hemisphere, closer to $\mathrm{P3}$; $\mathrm{P4}^{\prime}$, homologous of $\mathrm{P3}^{\prime}$ on the right hemisphere; $\mathrm{N1}, 50 \%$ of the distance on a line between $F 3$ and $T 3$ on the left hemisphere; N2, homologous of N1 on the right hemisphere; $\mathrm{N3}, 50 \%$ of the distance on a line between $\mathrm{P3}$ and $\mathrm{T3}$ on the left hemisphere; $\mathrm{N4}$, homologous of $\mathrm{N} 3$ on the right hemisphere; N5, midway on a line between the left preauricular depression and the left-eye canthus; N6, homologous of N5 on the right hemisphere; top left eye; top right eye; and nasion ( $1 \mathrm{~cm}$ above the nasion). 
For each subject...

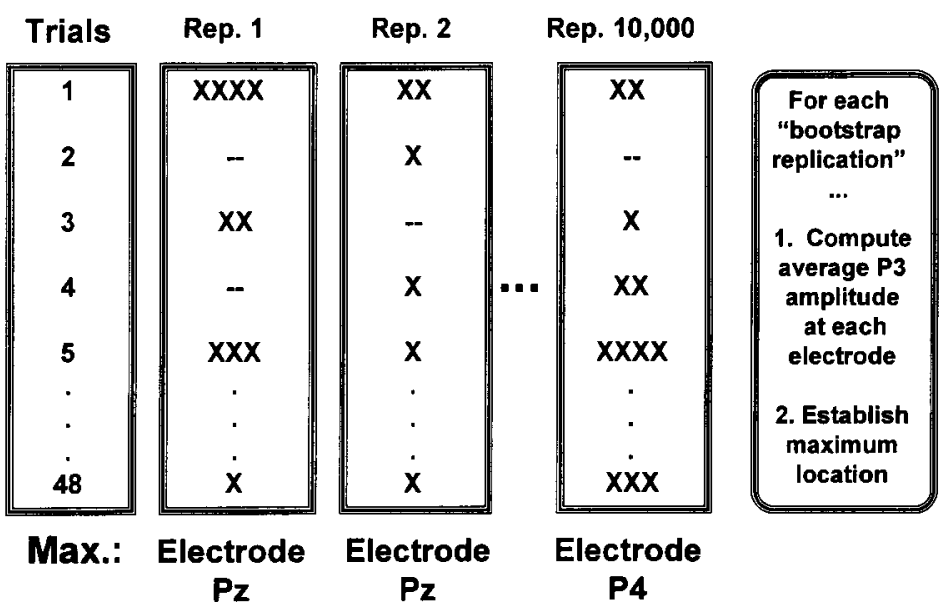

Figure 2. Schematic representation of the bootstrap procedure used for Example 1 . The $\mathrm{Xs}$ in the cells represent the number of times a particular trial is selected for a given bootstrap sample; the sign "-" indicates that a particular trial is not selected for that sample.

\section{Results}

A graphical representation of the frequency count data is presented in Figure 3. The data indicate that the bootstrap procedure allowed for a fast determination of the reliability of the locations of $\mathrm{P} 3$ maxima, as can be seen at a glance in Figure 3. The data appear to be fairly reliable for both young and old subjects. Even in cases in which the locations of the maxima were distributed over more than one electrode site, they tended to cluster within a set of adjacent electrodes. This latter result suggests that the actual maximum of the P3 may be located somewhere between the actual electrode sites and that the data may have been spatially undersampled. Greater spatial sampling may be useful in these cases (see Srinivasan, Tucker, \& Murias, 1998). In other cases, an interpolation technique (such as the spline interpolation; Perrin, Pernier, Bertrand, Giard, \& Echallier, 1987) may also be useful, provided that the analysis relates to a component that is widely distributed over the scalp (i.e., which has a low spatial frequency).

The high reliability of the scalp distributions of the individual subjects suggests that the old group may be a heterogeneous group, since individual differences in scalp distribution were observed (with some subjects showing target P3 maxima at frontal electrode sites and others at parietal electrode sites). These results contrasted with the homogeneity of the young group, in which all the subjects showed the typical posterior-maximum P3 distribution.

The bootstrap analysis described earlier provides information about the probability that the maximum of the P3 scalp distribution would be found at any particular electrode location. However, given that the bootstrap analysis is based on observed data that have an inherent variability, it is virtually certain that one location will have a larger frequency than all the others, even in the case in which no real differences in $\mathrm{P} 3$ amplitude exist among various electrode sites. Therefore, to evaluate the results of the bootstrap analysis, it is important to determine whether the observation that a particular location is more likely to yield the maximum P3 amplitude is due to the presence of a real maximum and not due to chance variations of the values. For this reason, a chi-square $\left(\chi^{2}\right)$ analysis was conducted on the distribution of frequencies identified by the bootstrap procedure. In performing this analysis, it is important to consider that the outcome of the bootstrap procedure does depend on the initial sample used. Since this sample was based on a limited

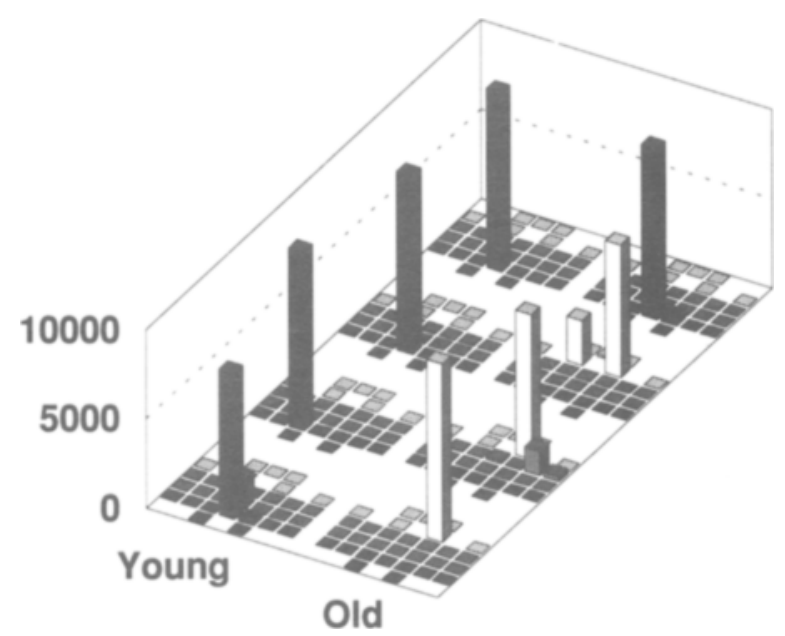

Figure 3. Example 1: ERP data. Three-dimensional plot of the frequency count distribution for 4 young subjects (left column) and 4 old subjects (right column). The locations are depicted according to the diagram presented in Figure 1. The shades from white to black represent a progression from anterior to posterior locations. 
number of observations (i.e., 48 trials per subject), the frequencies computed by the bootstrap analysis need to be normalized to the original number of observations used. In other words, the actual sample size is given by the number of trials actually recorded, and not by the number of bootstrap replications. In fact, the latter number can be increased arbitrarily. Since the $\chi^{2}$ is influenced by the number of cases, it is critical that the adjusted frequency counts be used (otherwise, significant $\chi^{2}$ values could be obtained for any subject and/or experiment). The normalized frequency is obtained using the following formula:

$$
F_{n}=F_{b} * \frac{N_{o}}{N_{b}}
$$

where $F_{n}$ is the "normalized" frequency for each cell, $F_{b}$ is the frequency obtained with the bootstrap analysis, $N_{o}$ is the number of cases (EEG trials) included in the sample used for the bootstrap analysis, and $N_{b}$ is the number of bootstrap replications.

The expected frequency for the $\chi^{2}$ is obtained from the hypothesis that no maximum target $\mathrm{P} 3$ amplitude exists and that there is an equal probability of observing a maximum at each of the electrode locations. Therefore, the expected frequency for each cell (electrode location) will be

$$
F_{e}=\frac{N_{o}}{N_{l}},
$$

where $N_{l}$ is the number of electrode locations used. The $\chi^{2}$ will then be computed using the following formula and will have $N_{l}-1$ degrees of freedom:

$$
\chi^{2}=\sum_{i=1}^{l} \frac{\left(F_{n_{i}}-F_{e}\right)^{2}}{F_{e}}
$$

The $\chi^{2}$ test will indicate the probability that the distribution of frequency values departs from that expected by chance. The probabilities of the $\chi^{2}$ for this example are shown in Table 1.

In addition to determining that the frequency of occurrence of P3 maximum is not equiprobable across electrode locations (as it would be if it were due to chance fluctuations of the values), it is also important to determine which locations have a greater-than-chance probability of being those with the maximum $\mathrm{P} 3$ amplitude. A conservative approach to this problem is to determine which is the minimum frequency count obtained with the bootstrap procedure for a particular electrode location that will contribute to the significance of an overall $\chi^{2}$ test. The frequency required for making the overall $\chi^{2}$ test significant can therefore be considered as a criterion level that will guide the interpretation of the bootstrap data. This criterion can be determined with the following formula:

$$
\text { criterion }=\frac{N_{b}}{N_{o}} *\left(F_{e}+\sqrt{F_{e} * \chi_{c}^{2}}\right),
$$

\begin{tabular}{|c|c|c|c|}
\hline Subject & $\begin{array}{c}\text { Electrodes } \\
\text { Exceeding } \\
\text { Criterion }\end{array}$ & $\chi^{2}(29)$ & $p$ \\
\hline \multicolumn{4}{|c|}{ Young Group } \\
\hline 1 & $\mathrm{Pz}$ & $1,392.00$ & $<.001$ \\
\hline 2 & $\mathrm{Pz}$ & $1,391.71$ & $<.001$ \\
\hline 3 & P3 & $1,389.99$ & $<.001$ \\
\hline 4 & $\mathrm{Pz}$ & 955.47 & $<.001$ \\
\hline \multicolumn{4}{|c|}{ Old Group } \\
\hline 1 & $\mathrm{Pz}$ & $1,266.40$ & $<.001$ \\
\hline 2 & Fp1, Fp2 & 825.36 & $<.001$ \\
\hline 3 & $\mathrm{Fp} 2$ & 921.77 & $<.001$ \\
\hline 4 & $\mathrm{Fp} 2$ & $1,387.11$ & $<.001$ \\
\hline
\end{tabular}

where $\chi_{c}^{2}$ is the $\chi^{2}$ associated with a $p=.05$ and with degrees of freedom equal to the number of electrode lo- cations minus 1 . In this example, the criterion is equal to $(10,000 / 48) *[1.6+$ SQRT of $(1.6 * 42.56)]$, which is equal to 2,053 (i.e., electrode locations with a frequency count exceeding this value in the bootstrap analysis are likely to contain an actual peak). Table 1 reports the electrode locations exceeding criterion for each of the subjects. For all subjects (except 1 ), only one electrode exceeded criterion.

\section{EXAMPLE 2 \\ Bootstrap Analysis of Peaks of the Event-Related Optical Signal (EROS)}

The event-related optical signal (EROS) is a newly developed technique whose purpose is to study the time course of neural activity in selected cortical areas (G. Gratton, Corballis, Cho, Fabiani, \& Hood, 1995; G. Gratton, Fabiani, et al., 1995). The dependent variable measured is the time required by near-infrared photons to travel through small areas of the head (photon delay). Photon delay increases (by a few picoseconds) when photons cross areas of the cortex that are active (for a review, see Fabiani, G. Gratton, \& Corballis, 1996) with respect to the same areas when they are inactive. This technique has a high temporal resolution, comparable to that of ERPs, and a good spatial specificity, being able to localize cortical activity to within a few millimeters from corresponding fMRI recordings (Fabiani et al., 1996; G. Gratton et al., 1997).

As is the case of ERPs, EROS is also a surface-projected measure, and surface maps of activity at particular latencies after stimulation can be constructed. These maps can have a spatial resolution of a few millimeters. However, given the individual variability in cortical anatomy, it can be expected that the maps obtained for the same experimental conditions in different subjects could vary considerably. For instance, since the exact location of the lower bank of the right calcarine fissure varies up to a few centimeters from one subject to another, the maximum effect obtained after $100 \mathrm{msec}$ from visual stimulation of the upper left quadrant of the visual field may actually occur at different locations in different subjects. For this 
reason, aggregation of the data from different subjects may not be appropriate, unless data can be first realigned on the basis of the individual subject anatomy. Therefore, it is very important to determine the reliability of the location of maximum of activity in each subject. In this example, we show how the bootstrap approach can be used for this purpose.

\section{Method}

Recording procedures. The stimulation and recording apparatus used to collect the data discussed here are described in detail in G. Gratton, Corballis, et al. (1995). A shortened description is given here. Three subjects were run in a visual stimulation experiment. Each subject was run for 12 sessions ( 1 for each of the scalp locations), each consisting of 48 blocks of trials. In each block, the subjects fixated at the center of a CRT monitor. Four black-and-white vertical grids were displayed continuously in each of the four quadrants of the monitor. In each block, one of the grids reversed (i.e., the black bars became white, and vice versa) at a rate of 2 per second. Each of these reversals was considered a stimulation. Depending on which of the grids reversed, four stimulation conditions were obtained: upper left, upper right, lower left, and lower right. Therefore, for each session, there were 12 blocks for each stimulation condition. Near-infrared (wavelength $=715 \mathrm{~nm}$ ) photons' delay was measured using a frequency-domain photon-migration method (E. Gratton et al., 1990) from 12 locations (corresponding to the different recording sessions) over the occipital area of the head, at a sampling rate of $20 \mathrm{~Hz}$. For each block, condition, location, and subject, the EROS response was obtained by averaging the individual traces following each of the 38 stimulations (i.e., each reversal). These preliminary "block averages" were used as individual cases for the bootstrap analysis. Averages for condition, location, and subject were then obtained. As shown by G. Gratton, Fabiani, et al. (1995; see also G. Gratton et al., 1997), the EROS response is characterized by a localized increase in photon delay, peaking $100 \mathrm{msec}$ after stimulation. The localization of this peak response varies depending on the stimulation condition, in a manner consistent with the contralateral-inverted representation of the visual field in primary visual cortex (i.e., it is maximum on the lower-left bank of area V1 for visual stimuli presented in the upper-right visual field, etc.).
Step 1: Creating the bootstrap samples. The purpose of the bootstrap analysis was to determine the reliability of the location of the maximum EROS and, in particular, of the location of the 100msec peak response. This was accomplished in the following way. The data used for this analysis were the amplitudes of the photon delay response at a latency of $100 \mathrm{msec}$ obtained for each block (12), location (12), condition (4), and subject (3). Only peak values were used for the analysis. The issue was whether the location exhibiting the average maximum value across the 12 blocks for each condition and subject was reliable. Therefore, for each condition and subject, 10,000 samples of the EROS peak response were obtained, each based on the average of 12 blocks sampled at random (with replacement) from the original sample of 12 recorded blocks (the bootstrap samples).

Steps 2 and 3: Bootstrap replications and data distribution. For each of these 10,000 samples, the location exhibiting the maximum value was determined. The frequency with which each location was selected as the maximum location was then tabulated. This frequency (divided by 10,000 ) would then be taken as the estimate of the probability that that particular location would have the highest value if the experiment were replicated 10,000 times. In the case of a null effect (i.e., when no peak is present), the expected probability for each cell is $1 / 12$, or .0833 , corresponding to a frequency of 833 out of 10,000 . This procedure is schematically illustrated in Figure 4.

\section{Results}

The results of the bootstrap procedure are shown in Table 2. A graphic representation of the results (for the upper-left and upper-right stimulation conditions only) is shown in Figure 5.

These data indicate that, for most stimulation conditions and subjects, some locations are much more likely than others to exhibit maximum photon delay values. This indicates some degree of localization of the EROS signal. In most cases, the locations with the highest frequencies have more than $50 \%$ probability of showing the maximum value when repeated samples are taken, and, in some cases, close to $100 \%$ probability. In general, the most likely lo-

For each subject and condition ...

\begin{tabular}{|c|c|c|c|c|}
\hline Block & Rep. 1 & Rep. 2 & Rep. 10,000 & \\
\hline 1 & $x x$ & $x$ & $X X X X X$ & \\
\hline 2 & $x$ & $\mathbf{x}$ & -- & $\begin{array}{l}\text { For each } \\
\text { "bootstrap" } \\
\text { replication: }\end{array}$ \\
\hline 3 & $\mathbf{x}$ & $x$ & $x$ & \\
\hline 4 & - & $x$ & $x x$ & $\begin{array}{l}\text { Find mean } \\
\text { photon delay }\end{array}$ \\
\hline 5 & $x x x$ & $x$ & $x \times x$ & \\
\hline - & - & - & - & 2. \\
\hline - & - & - & - & Establish \\
\hline 12 & $x$ & $x$ & $x$ & \\
\hline Peak: & Loc. B & Loc. B1 & Loc. $\mathbf{B 2}$ & \\
\hline
\end{tabular}

Figure 4. Schematic representation of the bootstrap procedure used for Example 2. The Xs in the cells represent the number of times a particular block is selected for a given bootstrap sample; the sign "-" indicates that a particular block is not selected for that sample. 
Table 2

Bootstrap Analysis of Location of the Maximum of the EROS Effects

\begin{tabular}{|c|c|c|c|c|c|c|c|}
\hline \multirow[b]{2}{*}{ Subject } & \multirow{2}{*}{$\begin{array}{c}\text { Vertical } \\
\text { Location } \\
\end{array}$} & \multicolumn{6}{|c|}{ Horizontal Location } \\
\hline & & Left & Midline & Right & Left & Midline & Right \\
\hline \multirow[t]{10}{*}{1} & & \multicolumn{3}{|c|}{ Upper Left } & \multicolumn{3}{|c|}{ Upper Right } \\
\hline & 2.5 & 5 & 0 & 64 & 84 & 0 & 63 \\
\hline & 2.0 & 5 & 6,570 & 2,506 & 0 & 1,249 & 20 \\
\hline & 1.5 & 0 & 39 & 664 & 1 & 8,555 & 13 \\
\hline & 1.0 & 145 & 0 & 2 & 1 & 14 & 0 \\
\hline & & \multicolumn{3}{|c|}{ Lower Left } & \multicolumn{3}{|c|}{ Lower Right } \\
\hline & 2.5 & 0 & 2 & 2,925 & 592 & 851 & 1,024 \\
\hline & 2.0 & 1 & 693 & 5,714 & 1,083 & 189 & 1,412 \\
\hline & 1.5 & 474 & 0 & 181 & 202 & 1,062 & 1,068 \\
\hline & 1.0 & 0 & 2 & 8 & 249 & 0 & 2,268 \\
\hline \multirow[t]{10}{*}{2} & & \multicolumn{3}{|c|}{ Upper Left } & \multicolumn{3}{|c|}{ Upper Right } \\
\hline & 2.5 & 733 & 455 & 1,395 & 433 & 2,434 & 1,540 \\
\hline & 2.0 & 1 & 483 & 0 & 1,887 & 0 & 273 \\
\hline & 1.5 & 1,153 & 1,657 & 4,065 & 807 & 2,441 & 11 \\
\hline & 1.0 & 32 & 21 & 5 & 155 & 8 & 11 \\
\hline & & \multicolumn{3}{|c|}{ Lower Left } & \multicolumn{3}{|c|}{ Lower Right } \\
\hline & 2.5 & 860 & 535 & 2 & 1,852 & 5,193 & 60 \\
\hline & 2.0 & 1,343 & 0 & 327 & 2 & 2 & 31 \\
\hline & 1.5 & 57 & 527 & 4,104 & 139 & 149 & 1,850 \\
\hline & 1.0 & 2,232 & 13 & 0 & 4 & 633 & 85 \\
\hline \multirow[t]{10}{*}{3} & & \multicolumn{3}{|c|}{ Upper Left } & \multicolumn{3}{|c|}{ Upper Right } \\
\hline & 2.5 & 0 & 33 & 7,847 & 18 & 971 & 413 \\
\hline & 2.0 & 5 & 882 & 13 & 1,869 & 5 & 66 \\
\hline & 1.5 & 0 & 3 & 3 & 0 & 2,887 & 510 \\
\hline & 1.0 & 85 & 1,129 & 0 & 571 & 2,687 & 3 \\
\hline & & \multicolumn{3}{|c|}{ Lower Left } & \multicolumn{3}{|c|}{ Lower Right } \\
\hline & 2.5 & 2 & 174 & 7,689 & 0 & 3,968 & 198 \\
\hline & 2.0 & 3 & 2 & 7 & 15 & 63 & 102 \\
\hline & 1.5 & 1 & 589 & 516 & 48 & 74 & 61 \\
\hline & 1.0 & 249 & 627 & 141 & 0 & 2,582 & 2,889 \\
\hline
\end{tabular}

Note-The locations indicated in bold are those that exceed the criterion $(p \leq .05)$.

cation of the maximum response for stimulation of the left portion of the visual field is to the right of the midline. In the case of stimulation of the right portion of the visual field, the most likely location of the maximum response is on the midline or to the left of midline, suggesting a slight asymmetry in the brain anatomy of the subjects participating in the study. Also, stimulation of the lower portion of the visual field is most likely to generate maxima that are at the same level or higher than those obtained with stimulation of the upper portion. Thus, in general, the data are consistent with the contralateral-inverted representation of the visual field.

The $\chi^{2}$ analysis was performed according to the procedures and formulas described for Example 1. The results are presented in Table 3 . The criterion for these data was 4,530 .

\section{DISCUSSION}

The two examples described in this paper indicate that a bootstrap approach can provide useful information about the reliability of the locations of maxima on the surface distribution of ERP and EROS data of individual subjects. Several types of inferences can be drawn. First, the bootstrap distribution provides an estimate of the probability that, if measures were replicated following the exact same procedures used for the original sample (and on the same subjects), a particular location would be found to be the maximum of the surface distribution. This probability value provides an indication of the confidence that can be placed in such statements as "the target P3 was maximum at the Pz electrode for subject X" or "the EROS response had its maximum $2.5 \mathrm{~cm}$ above and $1.5 \mathrm{~cm}$ to the left of the inion for subject Y."

The combination of the bootstrap procedure and simple, nonparametric tests, such as the $\chi^{2}$ test, can provide further insight about the data. An overall $\chi^{2}$ test can reveal whether the distribution of the bootstrap results is consistent with the hypothesis that all locations have an equivalent probability of being selected as the maximum. Therefore, it can test (and, if appropriate, reject) the hypothesis that no difference between locations exists.

It is also possible to analyze the contribution of each location to the overall $\chi^{2}$ and determine which locations (if any) are more likely than chance of having the maximum value. In this paper, we have presented a procedure to establish a criterion to evaluate the results of the bootstrap analysis that allows one to determine readily which 


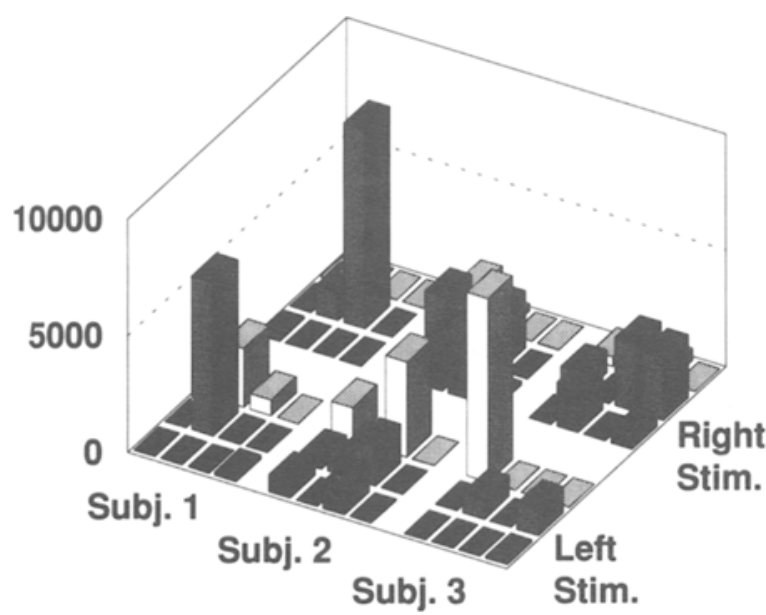

Figure 5. Example 2: EROS data. Three-dimensional plot of the frequency count distribution for the upper right (top row) and upper left (lower row) visual field stimulation in 3 subjects. Left locations are indicated by the color black, right locations by the color white.

locations these are. In the examples shown, the data indicated that all the locations of maxima of $\mathrm{P} 3$ amplitude and approximately $50 \%$ of those of EROS maxima were "significant" (i.e., were likely to be selected again in subsequent replications). This provides an estimate of the confidence level about the reliability of the location of the maximum value based on single-subject data.

These results indicate that bootstrapping is a conceptually simple methodology for analyzing surface distribution data, which circumvents the requirement of making a priori assumptions about the distribution of the data in the population. However, some limitations to its effectiveness should be kept in mind. First, the bootstrap procedure is inherently based on the sample of data that is used for the analysis. If this sample is, for any reason, not representative of the population it is intended to represent, the results of the bootstrap analysis will not be valid. Biases in data collection and systematic errors contained in the data will also be reflected in the results of the bootstrap procedure. Second, there are still several limitations in the availability of bootstrap software, although some programs for running bootstrap analyses are now available (e.g., Lunneborg, 1987; Peladeau \& Lacouture, 1993; see also freeware programs and infor-

Table 3

$\chi^{2}$ Test of Bootstrap Analysis of Location of Optical Maximum

\begin{tabular}{lcccccccc}
\hline & \multicolumn{2}{c}{ Subject 1} & & \multicolumn{2}{c}{ Subject 2} & & \multicolumn{2}{c}{ Subject 3 } \\
\cline { 2 - 3 } Stimulation & $\chi^{2}(11)$ & $p$ & & $\chi^{2}(11)$ & $p$ & & $\chi^{2}(11)$ & $p$ \\
\hline Upper left & 59.87 & $<.001$ & & 21.88 & $<.05$ & & 79.64 & $<.001$ \\
Upper right & 95.65 & $<.001$ & & 15.00 & n.s. & & 17.88 & n.s. \\
Lower left & 48.40 & $<.001$ & & 24.06 & $<.05$ & & 74.74 & $<.001$ \\
Lower right & 6.49 & n.s. & & 37.35 & $<.001$ & & 32.39 & $<.001$ \\
\hline
\end{tabular}

mation available on the World Wide Web ${ }^{1}$ ). Thus, it is likely that some ad hoc programming will be required.

Other investigators have proposed using bootstrapping or other distribution-free statistical methods for the analysis of ERP scalp distribution data. Karniski et al. (1994) proposed a permutation approach to determine the statistical significance of the difference between two topographic maps. This method is "distribution-free," because the results obtained with it do not depend on normality (or multinormality) assumptions. By contrast, with the bootstrap method, it is based on the computation of all possible combinations (permutations) of individual values. This allows for an "exact" computation of the probability of the observed pattern of data with respect to all possible samples. This method is impractical to use with a large number of subjects and/or trials. In the latter situation, the authors propose the use of a randomization test, quite similar in logic to the bootstrap procedure.

Srebro (1996) proposed using a bootstrap approach to address a slightly different problem from that described in the Karniski et al. (1994) paper-namely, to determine whether two scalp distributions are "identical" (rather than different). The method proposed by Srebro is logically very similar to the one reviewed here, although it is applied to a different problem in a between-subjects case, thus showing the flexibility of bootstrap methods.

In conclusion, we have presented two examples of the use of a bootstrap approach to analyze scalp distribution data and to determine the reliability of the location of maximum activity in individual subjects. The use of bootstrap methods is intended to supplement the variety of parametric statistical methods that have been suggested for analyzing surface distribution data (e.g., Brandeis \& Lehmann, 1989; Desmedt \& Chalklin, 1989; Hassainia, Petit, \& Montplaisir, 1994; McCarthy \& Wood, 1985; Naumann et al., 1992; Skrandies, 1989; Soufflet et al., 1991; Wong, 1991), rather than to replace them. With respect to parametric statistical methods, the bootstrap approach is more resistant to violations of assumptions-in particular, with respect to assumptions about the normality of the distribution. For this reason, it appears particularly appropriate for small sample sizes. There are several possible future developments for the application of bootstrap to the study of reliability. As mentioned earlier, the use of dense array recording techniques coupled with the assessment of confidence intervals may make this tool even more powerful.

\section{REFERENCES}

BRANDEIS, D., \& LEHMANN, D. (1989). Segments of event-related potential map series reveal landscape changes with visual attention and subjective contours. Electroencephalography \& Clinical Neurophysiology, 73, 507-519.

BrowN, J. K. (1994). Bootstrap hypothesis tests for evolutionary trees and other dendrograms. Proceedings of the National Academy of Sciences, 91, 12293-12297.

Chiano, M. N., \& Yates, J. R. (1994). Bootstrapping in human genetic linkage. Annals of Human Genetics, 58, 129-143. 
DeSMEDT, J. E., \& ChalKLIN, V. (1989). New method for titrating differences in scalp topographic patterns in brain evoked potential mapping. Electroencephalography \& Clinical Neurophysiology, 74, 359-366.

Diaconis, P., \& EFroN, B. (1983). Computer intensive methods in statistics. Scientific American, 248, 116-130.

Diner, B. C., Нolcom8, P. J., \& Dykman, R. A. (1985). P300 in major depressive disorder. Psychiatry Research, 15, 175-184.

DuFFY, F. H. (1982). Topographic display of evoked potentials: Clinical applications of brain electrical activity mapping (BEAM). In I. Bodis-Wollner (Ed.), Evoked potentials (Annals of the New York Academy of Sciences, Vol. 388, pp. 183-196). New York: New York Academy of Sciences.

EFron, B. (1979). Bootstrap methods: Another look at the jackknife Annals of Statistics, 7, 1-26.

EFroN, B., \& GoNG, G. (1983). A leisurely look at the bootstrap, the jackknife, and cross-validation. The American Statistician, 37, 36-48.

EFron, B., \& TiBShIRANI, R. (1985). The bootstrap method for assessing statistical accuracy. Behaviormetrika, 17, 1-35.

FABIANI, M., \& FrIEDMaN, D. (1995). Changes in brain activity patterns in aging: The novelty oddball. Psychophysiology, 32, 579-594.

Fabiani, M., Friedman, D., \& Cheng, J. C. (1998). Individual differences in P3 scalp distribution in old subjects, and their relationship to frontal lobe function. Manuscript submitted for publication.

Fabiani, M., Gratton, G., \& Corballis, P. M. (1996). Non-invasive NIR optical imaging of human brain function with sub-second temporal resolution. Journal of Biomedical Optics, 1, 387-398.

FABIANI, M., KaRIs, D., \& DonchIN, E. (1986). P300 and recall in an incidental memory paradigm. Psychophysiology, 23, 298-308.

FARWELl, L. A., \& DoNCHIN, E. (1988). Talking off the top of your head: A mental prosthesis utilizing event-related brain potentials. Electroencephalography \& Clinical Neurophysiology, 70, 510-523.

FORD, J. M., \& PFEFFERBAUM, A. (1985). Age-related changes in ERPs. In P. K. Ackles, J. R. Jennings, \& M. G. H. Coles (Eds.), Advances in psychophysiology (pp. 301-339). Greenwich, CT: JAI.

FRIEDMAN, D. (1995). Cognition in the elderly: An event-related potential perspective. In F. Boller \& J. Grafman (Eds.), Handbook of neuropsychology (pp. 213-240). Amsterdam: Elsevier.

FrIEDMAN, D., \& FABIANI, M. (1995). Memory and aging: An eventrelated brain potential perspective. In P. Allen \& T. Bashore (Eds.), Aging differences in word and language processing (pp. 345-389). Amsterdam: Elsevier.

Friedman, D., Simpson, G., \& Hamberger, M. (1993). Age-related changes in scalp topography to novel and target stimuli. Psychophysiology, 30, 383-396.

GevINS, A. (1996). Electrophysiological imaging of brain function. In A. W. Toga \& J. C. Mazziotta (Eds.), Brain mapping: The methods (pp. 259-276). San Diego, CA: Academic Press.

Gratton, E., Mantulin, W. W., van de Ven, M. J., Fishkin, J. B., Maris, M. B., \& Chance, B. (1990). The possibility of a nearinfrared optical imaging system using frequency domain methods. Third International Conference for Peace Through Mind/Brain Science (pp. 183-189). Hammamatsu City, Japan.

Gratton, G., Coles, M. G. H., \& Donchin, E. (1983). A new method for off-line removal of ocular artifact. Electroencephalography \& Clinical Neurophysiology, 55, 468-484.

Gratton, G., Corballus, P. M., Cho, E., Fabiani, M., \& Hood, D. C. (1995). Shades of gray matter: Noninvasive optical images of human brain responses during visual stimulation. Psychophysiology, 32, 505509.

Gratton, G., Fabiani, M., Corballis, P. M., Hood, D. C., Goodman, M. R., Hirsch, J., Kim, K., Friedman, D., \& Gratton, E. (1997), Fast and localized event-related optical signals (EROS) in the human occipital cortex: Comparisons with the visual evoked potential and fMRI. Neurolmage, 6, 168-180.

Gratton, G., Fabiani, M., Friedman, D., Franceschini, M. A., Fan- tini, S., Corballis, P. M., \& Gratton, E. (1995). Rapid changes of optical parameters in the human brain during a tapping task. Journal of Cognitive Neuroscience, 7, 446-456.

Hassainia, F., Petit, D., \& MontPlaisir, J. (1994). Significance probability mapping: The final touch in $t$-statistic mapping. Brain Topography, 7, 3-8.

KarIS, D., FABIANi, M., \& Donchin, E. (1984). P300 and memory: Individual differences in the von Restorff effect. Cognitive Psychology, 16, 177-216.

KARNISKI, W., Blair, R. C., \& SNIDER, A. D. (1994). An exact statistical method for comparing topographic maps, with any number of subjects and electrodes. Brain Topography, 6, 203-210.

LuNNEBorg, C. E. (1987). Bootstrap applications for the behavioral sciences. Educational \& Psychological Measurement, 47, 627-629.

MCCARTHY, G., \& Wood, C. C. (1985). Scalp distribution of eventrelated potentials: An ambiguity associated with analysis of variance models. Electroencephalography \& Clinical Neurophysiology, 62, 203-208.

Naumann, E., Huber, C., Maier, S., Plihal, W., Wustmans, A., Diedrich, O., \& BARTUSSEK, D. (1992). The scalp topography of P300 in the visual and auditory modalities: A comparison of three normalization methods and the control of statistical type II error Electroencephalography \& Clinical Neurophysiology, 83, 254-264

Peladeau, N., \& Lacouture, Y. (1993). SIMSTAT: Bootstrap computer simulation and statistical program for IBM personal computers. Behavior Research Methods, Instruments, \& Computers, 25, 410-413.

Perrin, F., Pernier, J., Bertrand, O., Giard, M. H., \& Echallier, J.F. (1987). Mapping of scalp potentials by surface spline interpolation. Electroencephalography \& Clinical Neurophysiology, 66, 75-81.

Pfefferbaum, A., Wenegrat, B., Ford, J. M., Roth, W. T., \& Kopell, B. S. (1984). Clinical application of the $P 3$ component of eventrelated potentials II: Dementia, depression and schizophrenia. Electroencephalography \& Clinical Neurophysiology, 59, 104-124.

Polich, J. (1991). P300 in the evaluation of aging and dementia. In C. H. M. Brunia, G. Mulder, \& M. N. Verbaten (Eds.), Event-related potential research (EEG Suppl. 42, pp. 304-323). Amsterdam: Elsevier.

PoLich, J., \& STARR, A. (1984). Evoked potentials in aging. In M. L. Albert (Ed.), Clinical neurology of aging (pp. 149-177). New York: Oxford University Press.

SKRANDIES, W. (1989). Data reduction of multichannel fields: Global field power and principal component analysis. Brain Topography, 2, 73-80.

SOUfFlet, L., Toussaint, M., Luthringer, R., Gresser, J., Minot, R., \& MACHER, J. P. (1991). A statistical evaluation of the main interpolation methods applied to 3-dimensional EEG mapping. Electroencephalography \& Clinical Neurophysiology, 79, 393-402.

SREBRO, R. (1996). A bootstrap method to compare the shapes of two scalp fields. Electroencephalography \& Clinical Neurophysiology, 100, 25-32.

SRinivasan, R., Tucker, D. M., \& Murias, M. (1998). Estimating the spatial Nyquist of the human EEG. Behavior Research Methods, Instruments, \& Computers, 30, 8-19.

ToGA, A. W., \& MazzlotTA, J. C. (EDs.) (1996). Brain mapping: The methods. San Diego, CA: Academic Press.

Wasserman, S., \& Bockenholt, U. (1989). Bootstrapping: Applications to psychophysiology. Psychophysiology, 26, 208-221.

WONG, P. K. (1991). Topographic representation of event-related potentials. Electroencephalography \& Clinical Neurophysiology, 42, 5-12.

\section{NOTE}

1. The following Web sites contain information about statistical software, including the bootstrap: http://gala.biom.cornell.edu/btry494 node50.html, http://lib.stat.cmu.edu/, http://www.astro.psu.edu/statcodes/, and http://www.stat.ucla.edu/ 